\title{
Apoptosis in the nervous system: New revelations
}

\author{
S-C Chen, T Curran, J I Morgan
}

\section{Introduction}

The elimination of neurones through programmed cell death is essential for the correct structural and functional maturation of the mammalian nervous system. ${ }^{1}$ Inappropriate neuronal death also occurs in a number of clinically important neuropathological states in the adult nervous system, such as stroke, amyotrophic lateral sclerosis, and Parkinson's and Alzheimer's diseases. Therefore, the elucidation of the mechanisms involved in apoptosis will contribute not only to our understanding of the normal development and physiology of the nervous system but also to its pathophysiology. Furthermore, the prevalence of apoptosis in the nervous system also suggests that brain tissue might be suitable for identifying and studying the specific molecular and cellular events that contribute to, or accompany, cell suicide.

A novel strategy for investigating neuronal death in the mammalian nervous system involves the use of transgenic mice carrying a fos-lacZ fusion gene. We have shown previously that a cellular immediate-early gene (cIEG) response precedes cell death in vivo and in culture. ${ }^{2}$ While the importance of the response itself is not entirely clear, monitoring of specific cIEG components in vivo provides a powerful anatomical mapping technique for detecting cells destined to die. As other methods of monitoring cIEG products are time-consuming or ambiguous, we have generated lines of transgenic mice in which a cIEG promoter drives expression of bacterial $\beta$-galactosidase. Thus, histochemical detection of $\beta$-galactosidase activity can be used to unambiguously monitor cIEG expression with single cell resolution either in fixed, frozen sections or in culture. This leader will discuss the background and application of cIEG mapping to the investigation of cell death in the adult and developing nervous system by highlighting results obtained with these transgenic mice.

\section{The immediate-early gene response}

Genes of the cellular immediate-early class, such as c-fos and c-jun, are characterised by their rapid induction in a protein synthesis independent manner. ${ }^{3}$ Many cIEGs encode transcription factors which are thought to serve as nuclear third messengers which constitute a link between extracellular stimuli and longer term alterations in gene expression. These inducible proteins control the expression of further, target, genes whose products contribute to an aspect of the cellular phenotype. The cIEG response is elicited by a diverse array of stimuli in many biological contexts, including mitogenesis, ${ }^{45}$ differentiation, ${ }^{67}$ neuronal activation, ${ }^{8-10}$ and even cell death. ${ }^{211-14}$ One can presume, therefore, that the target genes must be equally diverse in nature and precisely which ones are influenced by cIEG products must be dictated by the cell type involved, the stimulus, and its biological context.

Amongst the best characterised cIEG products are those encoded by the fos and jun proto-oncogenes. These genes belong to the basic-zipper superfamily of transcription factors which contain a leucine zipper, ${ }^{15}$ which facilitates protein-protein associations, and a region rich in basic amino acids which permits DNA binding. ${ }^{16}$ Members of the basiczipper superfamily form intra- and interfamily homo- and heterodimers via leucine zipper interactions. The various dimeric complexes then interact with specific DNA elements to bring about alterations in the transcription of target genes containing these elements-for example, Fos and Jun dimerise and bind to the activator protein 1 (AP-1) DNA consensus site (TGACTCA) which is essential for both basal and stimulated transcription of a series of genes. ${ }^{1718}$ Cellular IEGs are also members of the basic-zipper superfamily-for example, jun B is an inducible gene that is closely related to cjun. ${ }^{19}$ However, jun $\mathrm{D}$, another close family member of c-jun, is a constitutively expressed gene. ${ }^{20}$ Therefore, the cIEG response, viewed from the perspective of basic-zipper proteins, should be seen as one in which a variety of inducible transcription factors interact with each other and with resident transcription factors to bring about alterations in target gene expression.

Immediate-early gene expression as an anatomical mapping technique

As many types of stimuli induce a cIEG response, mapping the anatomical sites of expression of their products has become a popular approach for identifying the target cells upon which particular agents act in vivo. Furthermore, in the nervous system enhanced neuronal activity can elicit a cIEG response and so detection of cIEG products has even been used as a surrogate activity mapping technique much like 2-deoxyglucose uptake. Conventionally, immunohistochemistry or in situ hybridisation have been used to follow cIEG expression in tissue sections. These techniques, however, have certain drawbacks and limitations-for example, they can be time-consuming, they may not be amenable to extensive fine localisation analysis, in situ hybridisation does not detect protein, and

\author{
Correspondence to: \\ Dr J I Morgan. \\ Accepted for publication \\ 31 August 1994 \\ Molecular Biology, \\ Street, Nutley, \\ S-C Chen \\ T Curran \\ J I Morgan
}


immunohistochemistry frequently relies on commercial reagents which are notoriously variable and sometimes cross-react with related proteins. To circumvent these difficulties, a new technology has been pioneered in which a bacterial reporter gene, lacZ (which encodes $\beta$-galactosidase), is driven by a cIEG promoter. Such fusion genes have been introduced into the germline of mice and these transgenic animals now serve as a model in which $\beta$-galactosidase activity mirrors cIEG expression.

With respect to the c-fos-lac Z fusion gene, the construct was engineered such that it produced a fusion protein between Fos and lacZ, containing a sufficient contribution from Fos that it directed translocation of the fusion protein to the cell nucleus. ${ }^{21}$ Thus, $\beta$-galactosidase activity appears in the nuclei of cells in which c-fos-lacZ (and cognate c-fos) has been induced. A typical example of the
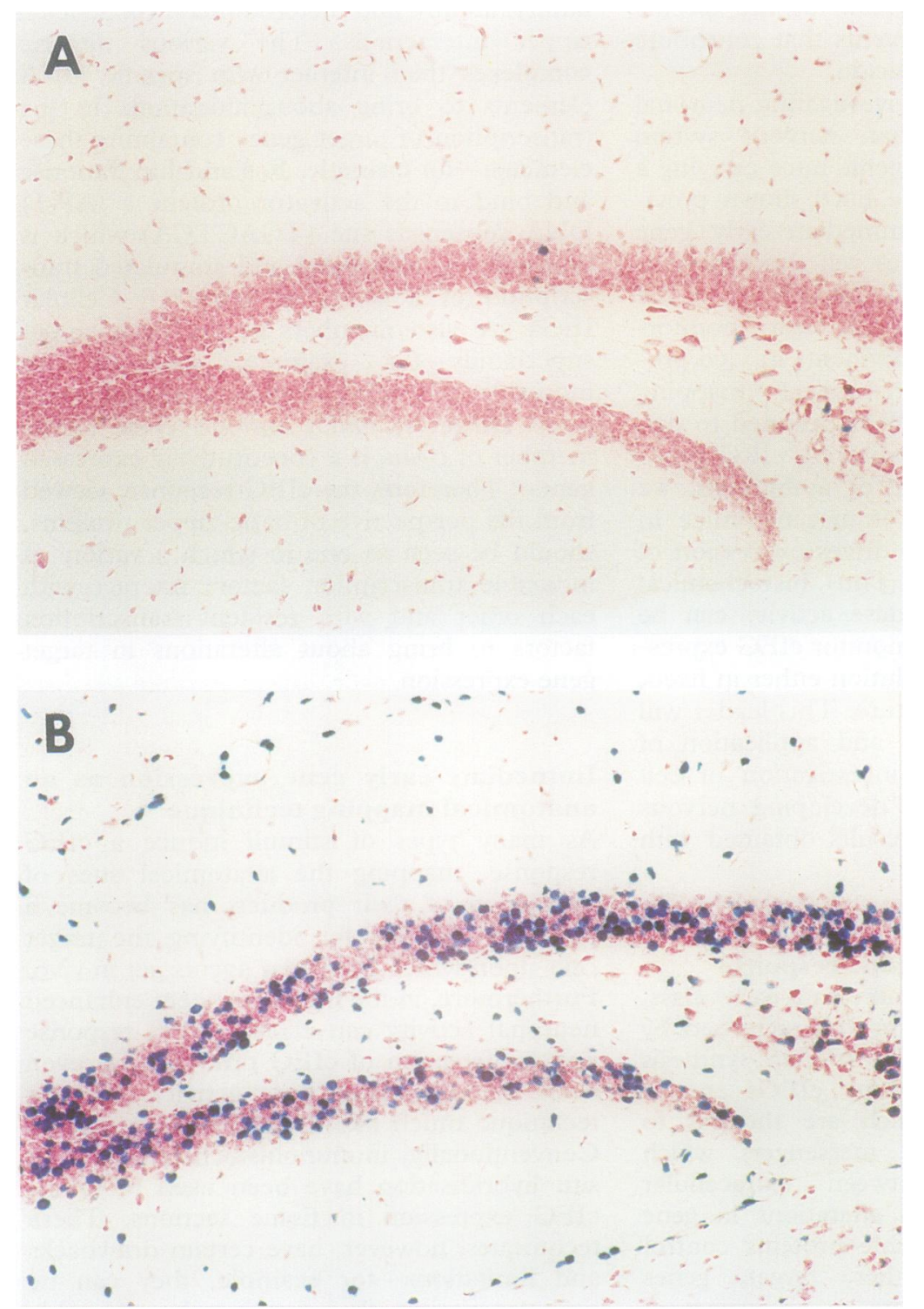

Induction of fos-lac $Z$ following kainic acid seizure. A: Very few Fos-lac $Z$ positive cells were detected in the dentate gyrus of the control transgenic animals. B: Two hours after kainic acid administration most of the cells in the dentate gyrus expressed Fos-lacZ. induction of the fos-lac $Z$ transgene in the nervous system is shown in the figure. The fusion gene also retains all of the known cisacting regulatory elements of c-fos as well as the $3^{\prime}$ untranslated region of the fos messenger RNA (mRNA) which is important for the stability of the fos message. ${ }^{22}$ In this configuration the kinetics of induction and repression of the transgene are similar to the cognate gene and both fos-lacZ mRNA and protein have short half-lives, like the endogenous gene products. Therefore, the net characteristics of the induction of the transgene and the longevity of its products largely reflect those of the endogenous gene.

\section{Fos-lacZ expression and neuronal death}

The conventional view is that c-fos expression is low in the unstimulated cell but rises transiently to quite high levels when an appropriate stimulus is applied. Generally, fos-lacZ expression fitted this profile, in that it was also undetectable in most tissues but could be induced with various types of challenges. ${ }^{21}{ }^{23}$ However, a number of instances were noted in which fos-lacZ expression was constitutively elevated in the adult transgenic mouse. Principal sites of continuous expression included the skin, hair follicles, bone, and nail bed. ${ }^{23}$ One common feature of these tissues is that the Fos-lacZ positive cells are all terminally differentiated and destined to die within a few days. This observation prompted us to examine other adult and fetal tissues and organs in which cell death occurs. This analysis revealed expression of fos-lac $Z$ in follicular cells of the degenerating ovarian follicles, interdigital web cells, and periderm cells of the embryo as well as in the heart valve cushion. ${ }^{24}$ These findings were the first suggestion that there might be a persistent cIEG response associated with the events leading to cell death.

In addition to the instances cited previously fos-lacZ expression was also observed in a number of neural structures at a time when there was active programmed cell death. Such instances included the hypoglossal and facial motor nuclei, the hippocampus, and superior cervical ganglion (unpublished observations, 1994, Smeyne et al). Sporadic Fos-lacZ positive neurones were also observed in the dorsal root ganglia and spinal motor neurons immediately before and during programmed cell death.

While the spatial and temporal expression of Fos-lacZ in the nervous system was consistent with the gene being expressed in neurones destined to die, it is difficult to be certain of the association. This is mainly because the number of Fos-lacZ positive cells per section is small as is the potential number of dying neurones. It should be remembered that neurones are selectively eliminated over several days and their corpses are phagocytosed within a few hours in the embryonic nervous system. ${ }^{1}$ Therefore, relatively few cells in any one section are dying and it is practically impossible to determine whether 
the Fos-lacZ positive cells represent the same small population of moribund neurones. However, the biology of the situation provides us with a means to increase the size of the dying population and to determine whether the number of Fos-lacZ positive cells increases in parallel.

Programmed cell death in the developing nervous system is largely used to match the sizes of two populations of cells-that is, it adjusts the size of the input population (which is usually produced in excess) to match the size of the target population. ${ }^{1}$ This excess of neurones may also provide for a certain degree of redundancy during development, permitting inadvertant cell loss. Mechanistically, this selection process often involves the competition of input neurones for trophic factors produced by the target cells or neurones. Typically, input neurones have a developmental window when their axons must encounter the trophic factor; neurones whose axons fail to enter their correct target field in time succumb to growth factor deprivation. Subsequently, neurones that have reached their targets form stable synapses and frequently lose their dependence on the trophic factor. This situation means that one can manipulate the fate of neurones by influencing their ability to obtain target derived growth factors. This can be achieved by simple surgical transection of the developing sciatic nerve, which separates the neurone from its target derived growth factors. If the surgery is performed in the neonatal transgenic mouse during the critical period, this results in the essentially synchronous suicide of ventral horn $\alpha$-motor neurones and sensory neurones in the dorsal root ganglia.

When the sciatic nerve was transected unilaterally in the neonatal fos-lac Z mouse there was an initial induction of the transgene in the entire body wall musculature and dorsal horn sensory neurones ipsilateral to the lesion. ${ }^{2}$ Subsequently, this expression subsided and 24 hours after surgery, Fos-lacZ expression appeared in the ipsilateral dorsal root ganglia and ventral motor neurones, where it persisted. This expression was not observed contralateral to the lesion and was restricted to the lumbar segments represented in the sciatic nerve. These are precisely the neuronal populations destined to die in this model. Thus, when the number of dying neurones in the sections is increased there is a parallel increase in the number of Fos-lacZ positive cells. Interestingly, if the same surgery is performed in the adult transgenic mouse, when the motor and dorsal root ganglion neurones have lost their dependency on target derived products, there is only a transient burst of Fos-lacZ expression (unpublished observations, 1994, Soares et al). Furthermore, other studies have indicated that in the adult the axotomised neurones show sustained expression of c-jun. ${ }^{25} 26$ Thus, continuous expression of c-jun may be an indicator of regeneration in the nervous system while persistent expression of c-fos may be a marker of neuronal demise.
From the molecular biological perspective these findings also indicate that c-fos and cjun are not always co-expressed, as has been inferred from their behaviour in cell culture. Indeed, in vivo each can be continuously expressed in the absence of the other. This raises several important issues. Firstly, the kinetics of expression prior to death (or regeneration) are quite unlike those seen in culture or in many other situations in vivo. Therefore, it is important that the molecular signalling events that induce this persistent pattern of expression be established as they may provide new clues to the secondary messenger pathways involved in cell death and regeneration. Secondly, the discordance of fos and jun expression raises the issue of which proteins interact with them in normal circumstances. Classically, transcription factor AP-1 was considered to be a heterodimer between Fos and Jun (or a Jun-Jun homodimer). In the degenerating neurone, however, this cannot be the case and in the regenerating neurone Jun may be dimerised with a protein other than itself. Clearly, it is now critical to identify the natural partners for Fos and Jun in these paradigms. One must also consider the intriguing possibility that the immediateearly response has a unique composition in these various natural situations and may contribute to these processes.

To pursue these issues further and to confirm that fos-lacZ expression is related to neuronal death, two types of experiments were performed. The first approach was to take advantage of the fact that there are inbred strains of mice that exhibit neuronal degeneration. ${ }^{27}$ In one of these mice strains, weaver, there is a degeneration of cerebellar granule cells during the first week after birth ${ }^{28}$ and a subsequent loss of neurones in the substantia nigra during adulthood. ${ }^{29}$ Therefore, the foslac $\mathrm{Z}$ mouse was crossed with the weaver strain and Fos-lacZ expression monitored in the neonatal cerebellum and adult substantia nigra. Both regions showed enhanced FoslacZ expression. Furthermore, in the cerebellum the Fos-lacZ positive cells were localised precisely to the regions where degenerating granule neurones were known to be. In the case of the cerebellum this is an example of an augmentation of programmed cell death, as granule cells are lost naturally during cerebellar development. ${ }^{30}$ The weaver mutation appears to greatly augment this process. In the substantia nigra, however, the situation is somewhat different in that it involves the delayed degeneration of neurones in the adult nervous system and is more akin to the human pathological condition, Parkinson's disease (for which weaver has been used as a model). Thus, Fos-lacZ appears to mark normal and augmented programmed cell death during development and slow neuronal degeneration in the adult. Whether this indicates that the two processes bear some molecular similarities is an intriguing point meriting further study.

The former strategy was used to demonstrate the coincidence of Fos-lacZ expression 
and cell death as well as providing a system in which molecular genetic and biochemical analyses can be performed. Besides programmed and genetically determined cell death, neurones may be killed by exposure to excitotoxins. ${ }^{31}$ These agents are typically direct or indirect agonists of glutamate receptors, the major excitatory neurotransmitter system in the mammalian brain. Several of the excitotoxins enter the food chain and pose substantial health risks to humans in some areas of the world. Administration of a typical excitotoxin, kainic acid, to mice or rats causes seizures followed, hours or days later, by the death of selective neuronal populations. In the case of kainic acid these neurons are found in the CA1 and CA3 fields of the hippocampus, the dorsomedial nucleus of the amygdala, and the pyriform cortex. ${ }^{32}$

After treatment of fos-lac $Z$ transgenic mice with kainic acid, the transgene was acutely induced in many regions of the nervous system. Twenty four hours after treatment, this expression had returned to baseline. Thus, this phase of fos-lac $Z$ expression appears to have been triggered by the seizures that immediately follow treatment with this, and other chemoconvulsants. Another convulsant agent, pentylenetetrazole (PTZ), also elicits seizures and a robust, but transient, induction of fos and fos-lacZ in many regions of the nervous system. ${ }^{923}$ However, in the kainic acid treated mouse fos-lac $Z$ expression is spontaneously re-induced in various neuronal cell populations days after administration of the excitotoxin. This second phase of expression is never seen with PTZ and occurs only in neuronal populations susceptible to kainic acid toxicity (for example, CA1 and CA3 pyramidal neurones of the hippocampus). One remarkable feature of this second period of expression is that $\beta$-galactosidase activity appears in the cytoplasm of vulnerable neurones, a situation never encountered in normal circumstances. This is taken to mean that these neurones may be degenerating and losing nuclear integrity permitting Fos-lacZ to leak into the cytoplasm. Alternatively, elevated proteolytic activity in compromised neurones may cleave galactosidase from the Fos sequences necessary for its translocation to the nucleus. Finally, this may be an indication of the failure, or overload, of the intracellular protein trafficking machinery. Whatever the explanation, the presence of a biphasic cIEG response and of cytoplasmic Fos-lacZ appears to be a striking marker of delayed neuronal death in vivo.

The finding of cytoplasmic Fos-lacZ associated with moribund neuronal populations provides a more refined marker with which to investigate the mechanisms involved in the demise of neurones following kainic acid treatment. As discussed earlier in this paper, cell death may be necrotic or apoptotic in nature. One biochemical feature frequently associated with apoptotic cell death is the active degradation of the genome into oligonucleosomal sized fragments, sometimes referred to as DNA ladders. ${ }^{33}$ This process can be monitored in tissue sections by the TUNEL (terminal transferase UTP nick end labelling) procedure which labels free 3' DNA terminal regions. ${ }^{34}$ Recently, it has been established that days after administration of kainic acid TUNEL positive neurones appear in vulnerable regions of the central nervous system. These TUNEL positive populations are coincident with cells with cytoplasmic Fos-lacZ staining (unpublished observations, 1994, Kasof et al). This leads us to suggest that kainic acid triggered neuronal death has features indicative of apoptosis and that Foslac $\mathrm{Z}$ expression is associated with this type of cellular demise. This suggestion is consistent with the coincidence of Fos-lacZ with neurones undergoing natural programmed cell death which is also believed to be apoptosis. The presence of apoptosis, rather than necrosis, is somewhat surprising as in culture glutamate and glutamate agonists rapidly kill neurones in a fashion resembling necrosisthat is, the cells swell up and appear to lyse, ${ }^{35}$ presumably as a consequence of the perturbation of ion (particularly calcium) balance. ${ }^{32}$ Swelling and lysis of intracellular membrane bound organelles are characteristic features of necrosis not normally associated with apoptosis. Therefore, to establish whether Fos-lacZ expression is uniquely associated with apoptosis it is important to determine whether it is expressed in cells undergoing necrotic death.

From the theoretical standpoint the overriding issue is the importance of the cIEG response in neuronal death. Is it involved in a causative manner? Is it an epiphenomenon? Is it a stress response aimed perhaps at counteracting the processes that lead to neuronal death?

The widespread distribution of Fos-lac $Z$ in terminally differentiating and dying cells suggests that the association of Fos expression with the impending demise of a cell is common to many cell types. Thus, this association can be observed naturally during development as well as in a number of tissues in the adult, such as skin, hair, and bone. Fos and Fos-lacZ expression is also triggered by events leading to unscheduled cell death. In addition to the examples elaborated above cIEG expression has been associated with cell death in the prostate following hormone deprivation ${ }^{11}$ and in various cell types following treatment with agents that damage DNA, such as topoisomerase inhibitors ${ }^{2}$ and ultraviolet irradiation. ${ }^{36}{ }^{37}$ In many of these examples, as in the kainic acid model, the expression of the transgene precedes the formal death of the cell by many hours or days. It is most unlikely, therefore, that the cIEG response is triggered by a catastrophic failure of intracellular signalling. Recent biochemical evidence also indicates that the composition of AP-1 complexes associated with moribund neurones is unique and quite unlike those found following seizures (unpublished observations, 1994, Kasof et al). This differential expression of cIEG products would not be compatible with random activation of the cIEG response by a collapse of cellular signalling pathways. 
While the induction of these genes before death may not be a trivial event, the association alone does not prove whether any of the gene products are actually involved in the process leading to cell death. The evidence supporting the direct involvement of cIEG products in cell death is derived from experiments where antisense oligonucleotides or transdominant suppressors have been introduced into cells in culture. ${ }^{12-14}$ In the case of fos and jun antisense oligonucleotides blocked apoptosis in lymphocytes. ${ }^{12}$ However, this was only evident when oligonucleotides to both gene products were used simultaneously, suggesting that the combined function of Fos and Jun is necessary for apoptosis following growth factor withdrawal in lymphoid cells. In the case of the steroid receptor NGFI-B, which is encoded by a cIEG induced in lymphoid cells before apoptosis, ${ }^{13} 14$ transdominant suppressors block death. ${ }^{13}$ Thus, this cIEG product also appears to be necessary for death to occur. Some caution is necessary when interpreting these experiments since inhibitors of protein and mRNA synthesis can block apoptosis and one needs to be sure that these treatments did not exert their action through non-specific inhibition of transcription or translation. However, there is also evidence from studies involving gene deletion which indicates that some of the cIEGs implicated in cell death are not necessary for apoptosis-for example, there is no overt disruption of programmed cell death in fos-null mice. ${ }^{38} 39$

Taken together, the case for the direct involvement of specific cIEGs in apoptosis is still unconfirmed. Therefore, an additional possibility should be considered. Namely, that the cIEG response is a reaction to the events that are set in motion by the cell suicide programme.

A scenario can be constructed in which some elements of the cIEG response are linked to oxidative stress or its consequences. This would account for the general induction of the cIEG response following seizures which result in increased metabolic demand in neurones, as manifested by increased 2 -deoxyglucose uptake. ${ }^{40}$ Indeed, maps of 2 '-deoxyglucose uptake are often coincident with the patterns of fos expression in the brain. ${ }^{40} \mathrm{~A}$ second feature of oxidative stress is the production of reactive oxygen intermediates that can damage macromolecules such as proteins, lipids, and DNA - events that have been implicated in neuronal death and glutamate toxicity. ${ }^{41}$ We and others have noted that free radical generating systems and DNA damaging agents, such as ultraviolet radiation, can induce a cIEG response. ${ }^{236} 3742$ Recent evidence also suggests that kainic acid leads to DNA damage in hippocampal neurones that express cytoplasmic Fos and Jun (unpublished observations, 1994, Kasof et al). Therefore, we would argue that this DNA damage could be the result of free radical damage triggered by the glutamate agonist.

The connection between oxidative stress, DNA damage, and cIEG expression is an intriguing one which may account for the presence of cytoplasmic Fos in vulnerable neurones. The binding of Fos-Jun heterodimers (as well as several other transcription factors) to DNA is subject to redox regulation. ${ }^{42}{ }^{43}$ The single cysteine residue conserved in all Fos and Jun family members must be constantly reduced to a sulphhydryl form for DNA binding to occur. ${ }^{42}$ Thus, loss of reducing capacity, as occurs during oxidative stress, would result in loss of DNA binding which might account for the leakage of Fos into the cytoplasm. A ubiquitous nuclear protein (Ref-1) has been cloned that carries out the reduction of the critical cysteine in Fos and Jun. ${ }^{44} 45$ Intriguingly, this enzyme has a dual activity in that it is also an apurinic/apyrimidinic endonuclease $\mathrm{H}^{46}$ - that is, a DNA repair enzyme responsible for dealing with bases damaged by free radicals. ${ }^{47}$ Thus, both the expression and biological activities of cIEG products appear to be controlled by oxidative stress and they may interact with the DNA repair system in neurones.

The next level of analysis must be directed at determining the role of individual cIEG products in the events leading to neuronal death in live animals. While the present transgenic animals provide useful correlates, future experiments will involve the generation of transgenic animals that produce genetically altered forms of cIEG proteins that potentiate or inhibit the function of the endogenous protein. Indeed, as we are dealing with a programme of gene induction it is likely that more than one product must be modified, or strategies evolved that simultaneously affect several genes. Finally, it will be interesting to establish whether cIEGs are expressed in the human brain in neuropathological conditions.

1 Oppenheim RW. Cell death during development of the nervous system. Annu Rev Neurosci 1991;14:453-501.

Smeyne RJ, Vendrell MV, Hayward M, Baker SJ, Miao GG, Schilling $\mathrm{K}$, et al. Continuous c-fos expression precedes programmed cell death in vivo. Nature 1993;363: 166-9.

3 Morgan JI, Curran T. Inducible proto-oncogenes of the nervous system: their contribution to transcription factors and neuroplasticity. Prog Brain Res 1990;86: 287-94.

4 Cochran BH, Reffel AC, Stiles CD. Molecular cloning of gene sequences regulated by platelet-derived growth factor. Cell 1983;33:939-47.

5 Curran T, Bravo R, Müller R. Transient induction of c-fos and $c-m y c$ in an immediate consequence of growth and c-myc in an immediate consequence of

6 Curran T, Morgan JI. Superinduction of fos by nerve growth factor in the presence of peripherally active benzodiazepines. Science 1985;229:1265-8.

7 Greenberg ME, Greene LA, Ziff EB. Nerve growth factor and epidermal growth factor induce rapid transient changes in proto-oncogene transcription in $\mathrm{PC} 12$ cells. f Biol Chem 1985;260:14101-10.

8 Morgan JI, Curran T. The role of ion flux in the control of c-fos expression: Nature 1986;322:552-5.

9 Morgan JI, Cohen DR, Hempstead JL, Curran T. Mapping patterns of c-fos expression in the central nervapping patterns of c-fos expression in the central

10 Hunt SP, Pini A, Evan G. Induction of c-Fos-like protein in spinal cord neurons following sensory stimulation. Nature 1987;328:632-4.

11 Buttyan R, Zaderi Z, Lockshin R, Wolgmuth D. Cascade induction of c-fos, c-myc, and heat shock $70 \mathrm{~K}$ transcripts during regression of the rat ventral prostate scripts during regression of the rat

12 Colotta F, Polentarutti N, Sironi M, Mantovani A. Expression and involvement of c-fos and c-jun protooncogenes in programmed cell death induced by growth factor deprivation in lymphoid cell lines. $\mathcal{F}$ Biol Chem 1992;267:18278-783. 
13 Woronicz JD, Calnan B, Ngo V, Winoto A. Requirement for the orphan steroid receptor Nur77 in apoptosis of Tcell hybridomas. Nature 1993;367:277-81.

14 Liu Z-G, Smith SW, McLaughlin KA, Schwartz LM, Osborne BA. Apoptotic signals delivered through the Tcell receptor of a T-cell hybrid require the immediatecell receptor of a T-cell hybrid require

15 Landschulz WH, Johnson PF, McKnight SL. The leucine binding zipper: a hypothetical structure common to a new class of DNA binding proteins. Science 1988;240 $1759-64$

16 Neuberg M, Schuermann M, Hunter JB, Müller R. Two functionally different regions in Fos are required for the sequence-specific DNA interaction of the Fos/Jun protein complex. Nature 1989;338:589-90.

17 Sassone-Corsi P, Sisson JC, Verma IM. Transcriptional autoregulation of the proto-oncogene fos. Nature 1988; 334:314-9.

18 Sonnenberg JL, Rauscher FJ 3rd, Morgan JI, Curran T. Regulation of proenkephalin by Fos and Jun. Science Regulation of pro

19 Ryder K, Lau LF, Nathans D. A gene activated by growth factors is related to the oncogene v-jun. Proc Natl Acad Sci USA 1988;85:1487-91.

20 Ryder K, Lanahan A, Perez-Albuerne E, Nathans D. jun D: a third member of the jun gene family. Proc Nat Acad Sci USA 1989;86:1500-3.

21 Schilling K, Luk D, Morgan JI, Curran T. Regulation of a fos-lacZ fusion gene: A paradigm for quantitative analysis of stimulus-transcription coupling. Proc Natl Acad S USA 1991;88:5665-9.

22 Curran T. Fos and Jun: intermediary transcription factors. In: Cohen P, Foulkes JG, eds. Molecular aspects of celluIn: Cohen P, Foulkes JG, eds. Molecular aspects of cellu-

23 Smeyne RJ, Schilling K, Robertson L, Luk D, Oberdick J, Curran $\mathrm{T}$, et al. Fos-lacZ transgenic mice: mapping sites of gene induction in the central nervous system. Neuron 1992;8:13-23.

24 Smeyne RJ, Schilling K, Oberdick J, Robertson L, Luk D Curran T, et al. A fos-lacZ transgenic mouse that can be used for neuroanatomic mapping. Adv Neurol 1993;59. 285-91.

25 Leah JD, Herdegen T, Bravo R. Selective expression of Jun proteins following axotomy and axonal transport block in peripheral nerves in the rat: evidence for a role in the regeneration process. Brain Res 1991;566: 198-207.

26 Jenkins R, Hunt SP. Long-term increase in the levels of cjun $\mathrm{mRNA}$ and Jun protein-like immunoreactivity in
motor and sensory neurons following axon damage. motor and sensory neurons

27 Caviness VS Jr, Rakic P. A mechanisms of cortical development: a view from mutations in mice. Annu $R e$ Neurosci 1978;1:297-326.

28 Smeyne RJ, Goldowitz D. Development and death of external granular layer cells in the weaver mouse cere bellum: a quantitative study. $\mathcal{f}$ Neurosci 1989;9: 1608-20.

29 Gupta M, Felten DL, Ghetti B. Selective loss of monoaminergic neurons in weaver mutant mice-an immunocytochemical study. Brain Res 1987;402. 379-82.
30 Vogel MW, Herrup K. Numerical matching in the mammalian CNS: lack of a competitive advantage of early Neurol 1989;283:118-28.

31 Choi DW. Excitotoxic cell death. I Neurobiol 1992;23: 1261-76.

32 Schwob JE, Fuller T, Price JL, Olney JW. Widespread patterns of neuronal damage following systemic or patterns of neuronal damage following systemic or intracerebral injections of kainic acid
study. Neuroscience 1980;5:991-1014.

33 Wyllie A, Morris RG, Smith L, Dunlop D. Chromatin cleavage in apoptosis: association with condensed chromatin morphology and dependence on macromolecular synthesis. $\mathcal{F}$ Pathol 1984;142:67-77.

34 Gavrieli Y, Sherman Y, Ben-Sasson SA. Identification of programmed cell death in situ via specific labeling of nuclear DNA fragmentation. f Cell Biol 1992;119: 493-501.

35 Coyle JT, Bird SJ, Evans RH, Gulley RL, Nadler JV, Nicklas WJ, et al. Excitatory amino acid neurotoxins: Nicklas WJ, et al. Excitatory amino acid neurotoxins: selectivity, specificity, and mechanis
Neurosci Res Prog Bulletin 1981;19:1-427.

36 Stein B, Rahmsdorf HJ, Steffen A, Litfin M, Herrlich P. UV-induced DNA damage is an intermediate step in UV-induced expression of human immunodeficiency virus type 1 , collagenase, $\mathrm{c}-\mathrm{fos}$, and metallothionein. $\mathrm{Mol}$ Cell Biol 1989;9:5169-81.

37 Devary Y, Gottlieb RA, Lau LF, Karin M. Rapid and preferential activation of the c-jun gene during the mammalian UV response. Mol Cell Biol 1991;11:2804-11.

38 Wang Z-Q, Ovitt C, Grigoriadis AE, Mohle-Steinlein U, Ruther U, Wagner EF. Bone and haematopoietic defects in mice lacking c-fos. Nature 1992;360:741-5.

39 Johnson RS, Spiegelman BM, Papaioannou V. Pleiotropic effects of a null mutation in the c-fos proto-oncogene. effects of a null mutati

40 Sharp FR, Gonzalez MF, Sharp JW, Sagar SM. c-fos expression and $\left({ }^{14} \mathrm{C}\right) 2$-deoxyglucose uptake in the caudal cerebellum of the rat during motor/sensory cortex stimulation. F Comp Neurol 1989;284:621-36.

41 Bondy SC, Lee DK. Oxidative stress induced by glutamate receptor agonists. Brain Res 1993;610:229-33.

42 Abate C, Patel L, Rauscher FJ 3d, Curran T. Redox regulation of Fos and Jun DNA-binding activity in vitro. Science 1990;249:1157-61.

43 Bannister AJ, Cook A, Kouzarides T. In vitro DNA binding activity of Fos/Jun and BZLF1 but not C/EBP is ing activity of Fos/Jun and BZLF1 but not C/EBP

44 Xanthoudakis S, Curran T. Identification and characterization of Ref-1, a nuclear protein that facilitates AP-1 zation of Ref-1, a nuclear protein that facilitates

45 Xanthoudakis S, Miao GG, Wang F, Pan Y-CE, Curran T. Redox activation of Fos-Jun DNA binding activity is mediated by a DNA repair enzyme. EMBO $f 1992$ 11:3323-35.

46 Xanthoudakis $S$, Miao GG, Curran $T$. The redox and DNA-repair activities of Ref- 1 are encoded by nonoverlapping domains. Proc Natl Acad Sci USA 1994;91: 23-7.

47 Doetsch PW, Cunningham RP. The enzymology of apurinic/apyrimidinic endonucleases. Mutat Res 1990; 236:173-201. 